\title{
Dielectric Microwave Absorbing Material Processed by Impregnation of Carbon Fiber Fabric with Polyaniline
}

\author{
Luiza de Castro Folgueras ${ }^{\mathrm{a}, \mathrm{b}}$, Evandro Luis Nohara ${ }^{\mathrm{b}}$, Roselena Faez, Mirabel Cerqueira Rezende ${ }^{\mathrm{d} *}$ \\ ${ }^{a}$ Comando-Geral de Tecnologia Aeroespacial - CTA, Instituto Tecnológico de Aeronáutica - ITA, \\ Praça Marechal-do-Ar Eduardo Gomes, 50, 12228-900 São José dos Campos - SP, Brazil \\ ${ }^{\mathrm{b}}$ Universidade de Taubaté, Rua Daniel Danelli, s/n, 12060-040 Taubaté - SP, Brazil \\ 'Universidade Federal de São Paulo, UNIFESP, Campus Diadema, 09972-270 Diadema - SP, Brasil \\ ${ }^{\mathrm{d} C o m a n d o-G e r a l ~ d e ~ T e c n o l o g i a ~ A e r o e s p a c i a l ~-~ C T A, ~ I n s t i t u t o ~ d e ~ A e r o n a ́ u t i c a ~ e ~ E s p a c ̧ o ~-~ I A E, ~}$ \\ Divisão de Materiais - AMR, Praça Marechal-do-Ar Eduardo Gomes, 50, \\ Vila das Acácias, 12228-904 São José dos Campos, SP - Brazil
}

Received: December 14, 2006; Revised: March 6, 2007

\begin{abstract}
It is a known fact that the adequate combination of components and experimental conditions may produce materials with specific requirements. This study presents the effect of carbon fiber fabric impregnation with polyaniline conducting polymer aiming at the radar absorbing material processing. The experiments consider the sample preparation with one and two impregnations. The prepared samples were evaluated by reflectivity measurements, in the frequency range of $8-12 \mathrm{GHz}$ and scanning electron microscopy analyses. The correlation of the results shows that the quantity of impregnated material influences the performance of the processed microwave absorber. This study shows that the proposed experimental route provides flexible absorbers with absorption values of the incident radiation close to $87 \%$.
\end{abstract}

Keywords: radar absorbing material, conducting polymers, dielectric absorber material, microwave

\section{Introduction}

The production of radar absorbing material (RAM) is directly related to the development of new materials for providing several applications involving tight requirements in the microwave reflection suppression. The electromagnetic pollution increase promoted by the rapid development of electronic and telecommunication systems has expanded in the form of electromagnetic interference ${ }^{1,2}$ (EMI), resulting in various studies involving the radar absorbing material technology. Aiming at controlling the problems created by EMI, the electromagnetic wave absorbing technology is an important topic to be considered for fulfilling civil and military purposes. Usually, in the military area the studies involve development of radar absorbing materials for the frequency range of 8 to $12 \mathrm{GHz}$. For this purpose, the role of RAM is to reduce target detection by the radar, i.e, to reduce its signature for radar detection (to reduce its eco-radar) ${ }^{3}$. For this, RAM is used to cover the surface of targets, which can consist of different types of equipment, land vehicles, aircraft and ships. In the radar absorbing material the processing of several parameters have to be taken into account. Among them, weight, thickness, microwave absorption, environmental resistance and mechanical strength are of major importance ${ }^{4,5}$.

Developing thin, flexible and light RAM is the main challenge of the studies involving microwave absorbers. For this, there are different processing routes, such as the impregnation of synthetic foams, fabrics and nonwoven substrates and manufacturing of paints and rubbers ${ }^{4}$.

The use of textile material as substrate or as reinforcement in the RAM processing is cited in the literature ${ }^{2,6,7}$ and has been under intensive research owing to its broad range of industrial applications. Conducting polymeric materials are currently being used in RAM processing, however, they can present certain problems related to adhesion, environmental stability and adequate values of electrical conductivity.

Innovations involving manufacturing procedures of RAM with conducting polymers are under study to improve the quality of this type of materials. For this, advancements are underway based on physicochemical analysis of the substrate surfaces and adhesion assessment of the conducting polymer, in order to increase both polymer doping degree and environmental stability ${ }^{8-10}$.

\subsection{Dielectric absorbers}

According to Balanis ${ }^{11}$, in dielectric materials the dominant charges of their atoms and molecules are positive and negative, which are kept in the same position by the atomic and molecular forces, and are not free to dislocate. However, upon applying an electric field to a dielectric material the formation of several electric dipoles takes place, which align themselves according to the orientation of the applied electric field. The reciprocal influence on the electric field causes the storage of electric energy, which can be turned to heating by Joule effect. And this phenomenon occurs with the dielectric absorbing materials.

The possible paths of an electromagnetic wave on a surface are shown in Figure 1, where the incident radiation can be absorbed, transmitted or reflected immediately or reflected back again (internal reflection). When an electromagnetic wave impinges on a surface coated with RAM, the incident radiation will not be totally absorbed immediately. The microwave attenuation by a RAM is significantly complex and different attenuation mechanisms can occur. Among the mechanisms involved in the microwave attenuation, the absorption of the incident energy and the multiple reflections of the radiation can be cited. In the former case, attenuation is promoted by dielectric 
or magnetic particles involving physical phenomena, such as ohmic losses and spin inversions, respectively. In the latter case, attenuation occurs by the backscattering of the radiation in different directions, using proper geometric shapes, which decreases the signal detected by the receptor. In this case, part of the incident radiation can create secondary waves, which can travel parallel to the surface, and when these waves find materials with different impedance values they cause internally reflected waves, improving the attenuation by the material ${ }^{8}$.

Equation $^{12,13}$, shows the components of the incident energy distribution inside the material, where $\mathrm{E}_{\mathrm{i}}, \mathrm{E}_{\mathrm{a}}, \mathrm{E}_{\mathrm{t}}$ and $\mathrm{E}_{\mathrm{r}}$ are the incident, absorbed, transmitted and reflected energies, respectively.

$$
\mathrm{E}_{\mathrm{i}}=\mathrm{E}_{\mathrm{a}}+\mathrm{E}_{\mathrm{t}}+\mathrm{E}_{\mathrm{r}}
$$

The attenuation is expressed according to Equation $2^{12,14}$, where $\mathrm{E}_{\mathrm{r}}$ and $\mathrm{E}_{\mathrm{i}}$ are reflected and incident energies, respectively. This parameter is expressed in $\mathrm{dB}$ (decibel).

$$
\text { Attenuation }(\mathrm{dB})=10 \cdot \log \frac{\mathrm{E}_{\mathrm{r}}}{\mathrm{E}_{\mathrm{i}}}
$$

Generally, absorbers are obtained by using different types of polymeric matrices, rigid or flexible, such as: epoxy, phenolic, bismaleimide, polyurethane, polyimide and silicone resins ${ }^{2}$.

The processing of a dielectric absorbing material is carried out by the adequate combination of absorbing centers (fillers) in a polymeric matrix, adjusting the dielectric characteristics to the application and the frequency range. Adequately adjusting the components during processing allows the material to change its behavior from transmitter or reflector to absorber.

In dielectric materials, the main properties that enable them to be applicable as microwave absorber are the dielectric constant and the dissipation factor of energy. Usually, the dielectric microwave absorbers change their dielectric properties through the thickness. This is normally achieved by the distribution of fillers that alters the electrical properties of the material ${ }^{2,15}$. For higher frequencies and higher dielectric constant values, greater reflected energy occurs from an untreated dielectric surface ${ }^{11,14,16,17}$.

Considering that both the air and the material dielectric constants are equal to 1 , no reflection is observed. In practice, the dielectric constants of non-metallic materials present high values ${ }^{2,18-21}$. Figure $2 \mathrm{a}$ illustrates the front face of a dielectric absorber, which demonstrates the surface impedance closely matching that one of the air. Under such condition, reflections from the surface are minimized. This case

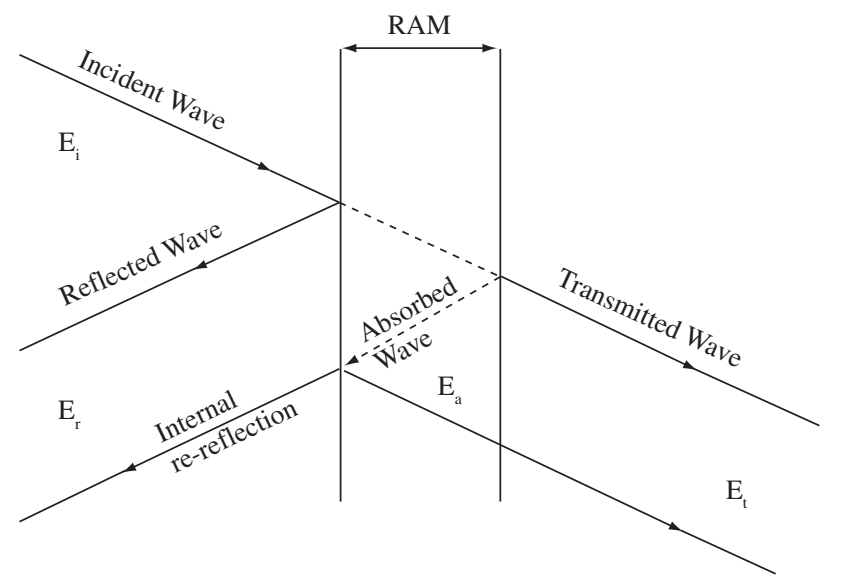

Figure 1. Summary of possible paths of incident electromagnetic wave in a material ${ }^{8}$. favors the incident wave propagation into the material, where the energy is dissipated and/or absorbed, characterizing the loss factor increase in the material. The impedance influence is an exponential function related to the thickness of the material ${ }^{2}$. In Figure $2 b$ the impedance decreases exponentially according to the proximity of the system's metal-support. In this case, it is important to emphasize that the existence of different layers in this material is directly related to the amount of absorbing center (filler) in each one. This adequate arrangement of layers can change the material behavior from a reflector to an electromagnetic radiation absorber.

This work deals with the preparation of dielectric absorbing materials by impregnation of carbon fabric substrate with polyaniline (PAni) added into a polyurethane matrix (PU), meeting the frequency range of 8-12 GHz. The carbon substrate was selected on account of its flexibility and lightness properties and its possibility for a wide range of applications in the aeronautical field. The absorbing performance was evaluated by reflectivity measurements and were correlated with morphological aspects of processed RAM.

\section{Experimental}

Carbon fabric style 4HS (4 Harness Satin Wave), supplied by Hexcel Composites, was used as substrate for the absorber processing. In the fabric configuration, the fill alternates one warp cable upwards and three warp cables downwards and the warp alternates one fill cable downwards and three fill cables upwards.

Polyaniline was chemically synthesized in lab scale at $0{ }^{\circ} \mathrm{C}$, by adaptation of the MacDiarmid method ${ }^{22}$. The polyaniline conducting polymer synthesis occurred based on the aniline oxidation, by using the ammonium peroxydisulfate $\left(\mathrm{NH}_{4}\right)_{2} \mathrm{~S}_{2} \mathrm{O}_{8}$ as oxidant agent in docecylbenzenesulfonic acid (DBSA). The oxidant agent was dropped slowly into the solution containing aniline monomer in DBSA medium. Stirring was carried out for 4-6 hours. This route produces the conducting polymer already doped - PAni (DBSA), i.e., the salt form of the polymer in the emeraldine oxidation state. The obtained powder was filtered, washed and dried at $60{ }^{\circ} \mathrm{C}$.

Afterwards, the doped PAni was added and dispersed in a bicomponent polyurethane matrix from Dexter Co., based on phthalic resin. This mixture was obtained in the concentration of $15 \%(\mathrm{w} / \mathrm{w})$ PAni (DBSA)/PU and the mixture's homogeneity was assured by mechanical mixing.

The application of the mixture on carbon substrate was prepared in different thicknesses by using conventional painting technique. The cure of PU followed a simplified cycle: 48 hours under air atmosphere,

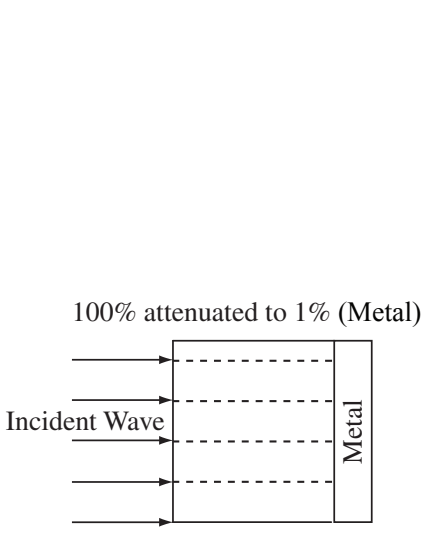

(a)

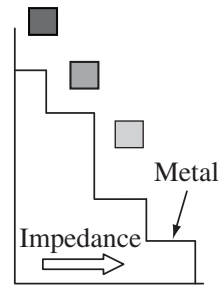

Combination of different materials

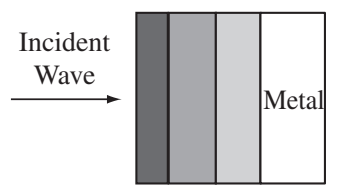

(b)

Figure 2. Schematic RAM showing the influence of a) thickness and b) multilayers on the wave attenuation ${ }^{2}$. 
at room temperature. This impregnation procedure allows several applications of PAni-PU layers, consequently, different thicknesses of the impregnated layer.

The prepared samples were evaluated by reflectivity measurements using the waveguide technique in a frequency range of 8 to $12 \mathrm{GHz}^{9,10,23,24}$. In order to guarantee precision of the measurements, the used setup was prepared with components presenting high mechanical precision. Basically, this system is formed by two coupled waveguides, one straight and another curve fitted to a HP scalar spectrum analyzer, model 8757A and an Agilent synthesized sweeper generator, model 83752A, as shown in Figure 3. An aluminum plate was used as reference material with $0 \%$ of attenuation or $100 \%$ reflector. This material is considered a perfect reflector.

The morphology of the prepared samples was observed by scanning electron microscopy (SEM) by means of a LEO equipment model $435 \mathrm{Vpi}$, without special sample preparation.

\section{Results and Discussion}

Figure 4 shows the aspect of the impregnated and non-impregnated carbon fabric substrate. The samples obtained by painting present one side of the fabric more impregnated than the other, consequently, a heterogeneous distribution of the conducting polymer into the carbon fabric faces is observed.

The impregnated carbon fabric has evidenced important characteristics, such as flexibility, low density $\left(<1 \mathrm{~kg} / \mathrm{m}^{2}\right)$ and easy-handling. By comparing the density values of the processed dielectric material with the conventional magnetic absorbers based on ferrites (density of $4-5 \mathrm{~kg} / \mathrm{m}^{2}$ absorbing around $10 \mathrm{~dB}$ ), it is substantiated that the studied RAM is nearly 4-5 times lighter ${ }^{2}$.

Figure 5 shows the surface morphology of the impregnated samples. A good wetting of the carbon fiber fabric is observed, thus providing an adequate anchorage of the PAni-PU mixture into the substrate. SEM images confirm that the impregnation occurs preferably on one face of the carbon fabric substrate. The good anchorage

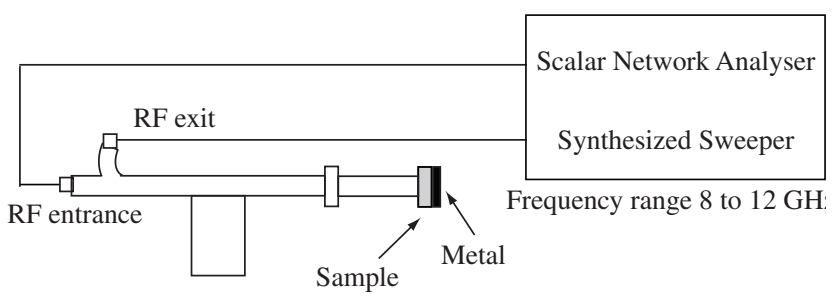

Figure 3. Schematic reflectivity measurement setup.

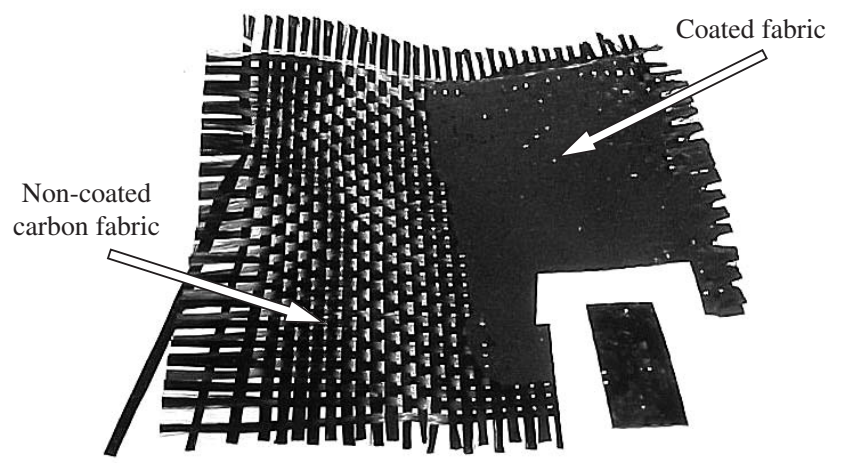

Figure 4. Carbon fabric substrate impregnated with doped PAni - PU. is also observed during handling of the sample, where material loss is not verified.

Figure 6 represents the external surface of the impregnated sample. Figure $5 \mathrm{~b}$ shows the upper face of the sample revealing rich regions of PAni-PU and polyaniline particles distributed in the polyurethane matrix, forming conducting regions (thickness of approximately $1.5 \mathrm{~mm}$ ).

According to the SEM images it is observed that the polyurethane matrix supported the conducting polymer particles, hence favoring the contact among them. This characteristic is necessary to promote the electrical conductivity and, probably, the absorption of the electromagnetic energy in the material. The anchorage of the particles was also improved by the texture of the carbon fiber fabric.

Figure 7 shows the reflectivity curve of the processed dielectric material based on PAni-PU carbon fabric with one and two layers. The horizontal black line shows the characterization of the carbon fabric without impregnation positioned over an aluminum plate (100\% reflector or $0 \%$ attenuation - metallic reference). It is observed that the carbon fabric presents reflector behavior, similar to that one observed for the aluminum plate. It is also observed that the number of impregnations (different layers) assigns distinct behaviors of electromagnetic radiation attenuation by the prepared samples.

The material with one layer of impregnation presents low values of attenuation, nearly $1 \mathrm{~dB}$ close to the reference curve; this result suggest that the material processed with one layer is inadequate to be



(a)

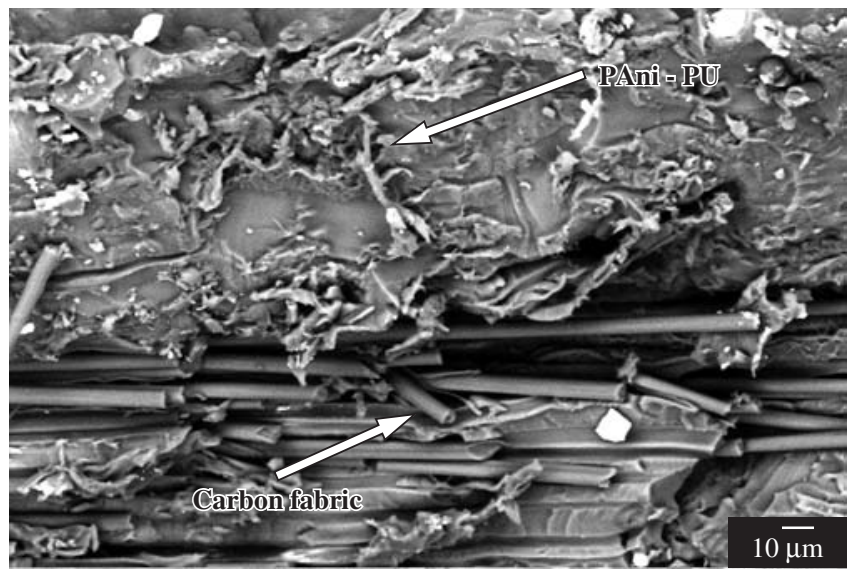

(b)

Figure 5. SEM of the substrate impregnated with PAni-PU: a) lateral edge view of the sample; and b) detailed view of the carbon fiber wetting. 


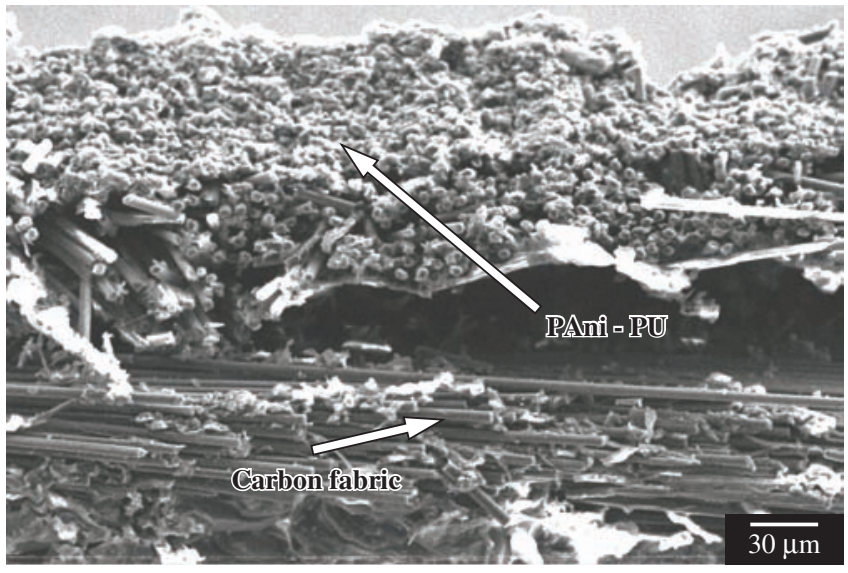

(a)

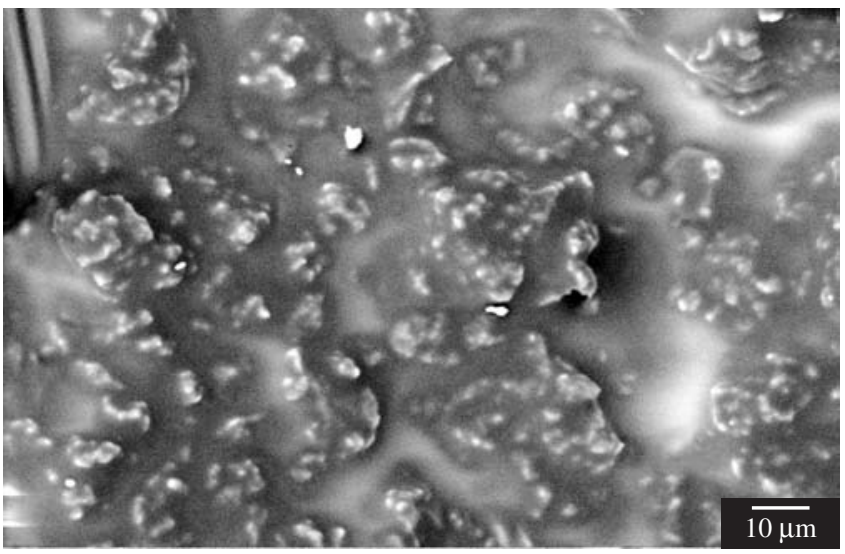

(b)

Figure 6. SEM: a) cross section, showing the thickness of the impregnated layer; and b) impregnated layer.

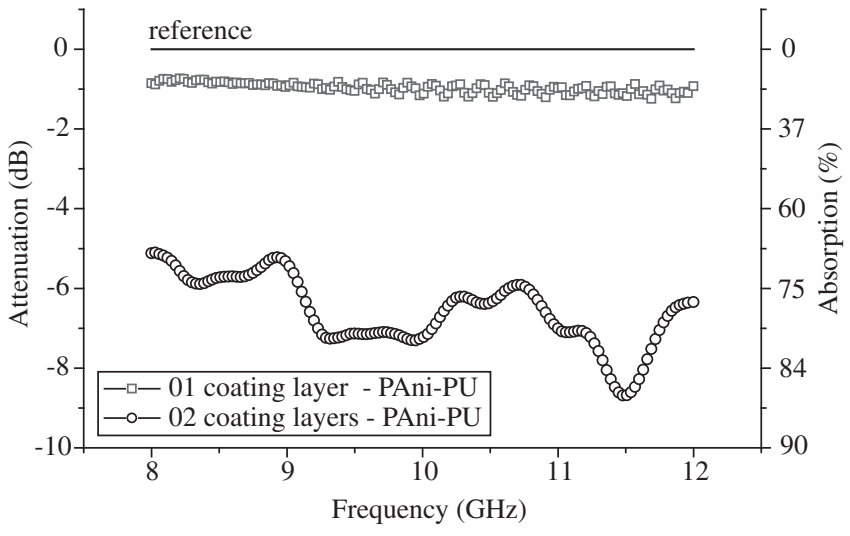

Figure 7. Reflectivity curves of the material based on PAni-PU impregnated in carbon fabric substrate.

used as radar absorbing material. The observed behavior is attributed to the heterogeneity of the impregnation and the rough aspect of the carbon fiber fabric.

The sample with two impregnations shows higher values of attenuation in the frequency range of 8-12 GHz. At the frequency of $8 \mathrm{GHz}$ the attenuation value is close to $5 \mathrm{~dB}$, which corresponds to $68 \%$ of the absorbed energy ${ }^{25,26}$. From this point it is observed that attenuation increases with medium values of $7 \mathrm{~dB}$ between 9 and $10 \mathrm{GHz}$ (nearly $80 \%$ of absorption) and a maximum value of $9 \mathrm{~dB}$ at $11.5 \mathrm{GHz}$ ( $87 \%$ of absorption of the incident energy). In a general manner, this sample shows broadband absorber behavior. This behavior is an uncommon characteristic for absorber composites based on polyaniline presenting narrow absorber behavior, according to the literature ${ }^{7,15}$. In this study, such characteristic is attributed to the texture of the carbon fiber fabric that also favored losses by multiple scattering. This is an interesting result for different applications in EMI, considering the flexibility of the obtained material, the thermal stability of the carbon support and the larger frequency range of the processed radar absorbing material.

This result suggests that the absorbing characteristic is directly related to the amount of conducting polymer in the substrate, which can change the conductivity and, consequently, the absorption of the incident radiation. Another parameter to be considered is the thickness of the absorbing layer.
Another consideration is in relation to the reflector behavior of the carbon fabric, which allows to be used as reflector in different applications (for example, in antenna reflectors), presenting the same behavior of aluminum plates, as shown in Figure 6 and mentioned in literature ${ }^{2}$. However, this work shows that the reflector behavior can be changed to the absorber one by adequate application of a conducting coat, for example, a PAni-PU mixture.

\section{Conclusions}

The dielectric material prepared in this work presented proper characteristics for handling, flexibility and lightweight, meeting requirements for its application in complex-shaped artifacts. Other important characteristic of the processed RAM include its low specific mass (less than $1 \mathrm{~kg} / \mathrm{m}^{2}$ ).

The correlation of SEM observations and the reflectivity results enabled concluding that the processed material presented good wetting of carbon fabric substrate by the PAni mixture, which favored manufacturing of an absorber that attenuates nearly $80 \%$ of the incident radiation, in the frequency range of $8-12 \mathrm{GHz}$. This result shows the viability of the broadband RAM processing with the proposed components (carbon fiber fabric, PAni and PU resin).

\section{Acknowledgments}

The authors acknowledge CNPQ for the financial support (Project numbers: 303528/2003-6 and 151929/2005-0).

\section{References}

1. Yusoff AN, Abdullah MH, Mansor AA, Hamid SAA. Electromagnetic and absorption properties of some microwave absorbers. Journal of Applied Physics. 2002; 92(2):876-880.

2. Lee SM, editor. International Encyclopedia of Composites. New York: VHC Publishers, 1991

3. Nicolaescu I, Oroian T. Radar Cross Section. In: Telecommunications in Modern Satellite, Cable and Broadcasting Service. TELSIKS 2001. Proceedings of the fifth International Conference on; 2001 Sep 19-21; Nis, Yugoslav. 2001. p. 65-68.

4. Jung-Hoon Oh, Kyung-Sub Oh, Chun-Gon Kim, Chang-Sun Hong. Design radar absorbing structures using glass/epoxy composite containing carbon black in the $\mathrm{X}$ frequency ranges. Composites Part B: Engineering. 2004; 35(1):49-56.

5. Antar YMM, Liu HW. Effect of radar absorbing materials on RCS of partially coated targets. Microwave and Optical Technology Letters. 1998; 17(5):281-284. 
6. Chawla, KK. Composite materials science and engineering. 2. ed. New York: Springer-Verlog, 1998.

7. Olmedo L, Hourquebie P, Jousse F. Microwave Properties of Conductive Polymers. In: Nalwa HS, editor. Handbook of Organic Conductive Molecules and Polymers. U S: John Willey \& Sons, 1997.

8. Dhawan SK, Singh N, Venkatachalam S. Shielding effectiveness of conducting polyaniline coated fabrics at $101 \mathrm{GHz}$. Synthetic Metals. 2002; 125(3):389-393.

9. Nalwa HS, editor. Handbook of Organic Conductive Molecules and Polymers. Chichester: John Wiley Sons, 1997.

10. Folgueras LC. Obtenção e caracterização de materiais absorvedores de microondas flexíveis impregnados com polianilina. [Tese de Doutorado]. São José dos Campos, Brazil: Instituto Tecnológico de Aeronáutica; 2005.

11. Balanis CA. Avanced Engineering Eletromagnetics. New York: John Wiley Sons, 1989.

12. Epstein AJ, Joo J. Electromagnetic radiation shielding by intrinsically conducting polymers. Applied Physics Letters. 1995; 65(18):2278-2280.

13. Huang JC. EMI Shieldind plastics: a review. Advances in Polymer Technology. 1995; 14(2):137-150.

14. Knott, EF.; Shaeffer, JF.; Tuley, MT. Radar cross section: Its prediction, measurement and reduction. Dedham Massachusetts: Artech House, Inc., 1985.

15. Hourquebie P, Blondel B, Dhume S. Microwave and optical properties of soluble conducting polymers. Synthetic Metals. 1997; 85(1-3):1437-1438.

16. Folgueras LC, Faez R, Rezende MC. Wave guide characterization of flexible absorbing material. In: Microwave and Optoelectronics Conference;
2003. IMOC 2003. Proceedings of 2003 SBMO/IEEE MTT-S International; 2003 Sep 20-23; Foz do Iguaçu, Brazil. 2003. p. 741-745.

17. Hourquebie $P$, Olmedo L. Influence of structural parameters of conducting polymers on their microwave properties. Synthetic Metals. 1994; 65(1):19-26.

18. Olmedo L, Hourquebie P, Jousse F. Microwave properties of conductive polymers. Synthetic Metals. 1995; 69(1-3):205-208.

19. Rmili H, Miane J-L, Zangar H, Olinga TE. Microwave conductivity measurements of high conductive polyaniline films. The European Physical Journal Applied Physics. 2005; 29(1):65-72.

20. Quéffélec P, Le Floc'h M, Gelin P. Broad-band characterization of magnetic and dieletric thin films using a microstrip line. IEEE Transactions on Instrumentation and Measurement. 1998; 47(4):956-963.

21. Curri S, Tran VH. The electromagnetic properties of poly(p-phenylenevinylene) derivatives. Polymer. 1998; 39(12):2399-2408.

22. MacDiarmid AG, Chiang JC, Richter AF, Somarisi NLD. In: Alcacer L, editor. Conducting Polymers: Special Applications. Reidel Dordrecht, 1987.

23. Miacci MAS. Medidas de seção reta radar de alvos de geometrias complexas na faixa de 8 a 12 GHz. 2002. [Dissertação de Mestrado]. São José dos Campos, Brazil: Instituto Tecnológico de Aeronáutica; 2002.

24. Rezende MC, Silva FS, Martin IM. Materiais absorvedores de radiação eletromagnética. Spectrum. 2000; 2:17-20.

25. Barton DK, Leonov SA. Radar Technology Encyclopedia. London: Artech House, 1997.

26. Laverghetta TS. Microwave Measurements and Techniques. Dedham Massachusetts: Artech House, Inc., 1976. 
